\title{
Transcription factor TEAD4 regulates expression of Myogenin and the unfolded protein response genes during $\mathrm{C} 2 \mathrm{C} 12$ cell differentiation
}

\author{
A Benhaddou ${ }^{1}$, C Keime ${ }^{1}$, T Ye ${ }^{1}$, A Morlon ${ }^{1}$, I Michel ${ }^{1}$, B Jost ${ }^{1}$, G Mengus ${ }^{1}$ and I Davidson ${ }^{*, 1}$
}

The TEAD (1-4) transcription factors comprise the conserved TEA/ATTS DNA-binding domain recognising the MCAT element in the promoters of muscle-specific genes. Despite extensive genetic analysis, the function of TEAD factors in muscle differentiation has proved elusive due to redundancy among the family members. Expression of the TEA/ATTS DNA-binding domain that acts as a dominant negative repressor of TEAD factors in $\mathrm{C} 2 \mathrm{C} 12$ myoblasts inhibits their differentiation, whereas selective shRNA knockdown of TEAD4 results in abnormal differentiation characterised by the formation of shortened myotubes. Chromatin immunoprecipitation coupled to array hybridisation shows that TEAD4 occupies 867 promoters including those of myogenic miRNAs. We show that TEAD factors directly induce Myogenin, CDKN1A and Caveolin 3 expression to promote myoblast differentiation. RNA-seq identifies a set of genes whose expression is strongly reduced upon TEAD4 knockdown among which are structural and regulatory proteins and those required for the unfolded protein response. In contrast, TEAD4 represses expression of the growth factor CTGF (connective tissue growth factor) to promote differentiation. Together these results show that TEAD factor activity is essential for normal $\mathrm{C} 2 \mathrm{C} 12$ cell differentiation and suggest a role for TEAD4 in regulating expression of the unfolded protein response genes.

Cell Death and Differentiation (2012) 19, 220-231; doi:10.1038/cdd.2011.87; published online 24 June 2011

The TEAD transcription factors make a highly conserved family of 4 DNA-binding proteins ${ }^{1,2}$ containing the TEA (Yeast (TEC-1), Aspergillus nidulans (AbaA) and Drosophilla (scalloped))/ATTS (Aspergillus nidulans (AbaA), Yeast (TEC-1), human TEF1, and Drosophilla (scalloped)) DNA-binding domain (DBD). ${ }^{3,4}$ The TEA domain comprises a three-helix bundle with a homeodomain fold and binds a consensus MCAT (5'-CATTCCA $\mathrm{T}-3^{\prime}$ ) element originally defined as the GT-II motif of the simian virus 40 (SV40) enhancer. ${ }^{5}$ Mammalian TEADs are widely expressed with prominent expression in the nervous system and muscle. In-vitro, cell-based, knockout and transgenic studies have addressed the role of TEAD factors in regulation of muscle-expressed genes. ${ }^{6-8}$ Cardiac troponin $\mathrm{T}$, myosin, heavy polypeptide 7 , cardiac muscle, beta $(\beta-\mathrm{MHC})$ and Myocardin, have functional MCAT motifs in their regulatory regions. $^{2}$ Stimulation of $\alpha 1$-adrenergic signalling has been shown to induce cardiac hypertrophy and activate transcription of the $\beta$-MHC and skeletal $\alpha$-actin genes in an MCAT- and TEAD-dependent manner in cultured neonatal rat cardiomyocytes. ${ }^{9}$ Cardiac muscle-specific overexpression of TEAD4 in transgenic mice has been shown to induce arhythmias in vivo. ${ }^{8}$

TEAD4 is specifically expressed in developing skeletal muscle in mouse embryos. ${ }^{1}$ Chromatin immunoprecipitation-array hybridization (ChIP-chip) showed that TEAD4 is a direct target of the MYOD1 and MYOG transcription factors in C2C12 cells. ${ }^{10}$ Although TEAD4 upregulation by MYOD1 and MYOG during differentiation is thought to activate transcription of muscle structural genes, mouse knockouts do not show any evident role for TEAD4 in muscle development. TEAD4 knockout leads to lack of trophectoderm specification and early preimplantation lethality. ${ }^{11}$ Conditional TEAD4 inactivation shows that it is not required for post-implantation development perhaps due to redundancy with other members of the family.

TEAD factors also mediate control of proliferation and organ size via the Hippo pathway in both Drosophila and mammalian cells. ${ }^{12}$ The TEAD C-terminal region interacts with the Yes-associated protein 1 (YAP1) and TAZ/WWTR1 (WW domain containing transcription regulator 1) coactivators that are phosphorylated and inhibited by the Hippo tumor suppressor pathway. ${ }^{12}$ TEADs are required for YAP-induced cell growth, oncogenic transformation, and epithelialmesenchymal transition via activation of connective tissue growth factor (CTGF) expression.

The above results suggest that TEADs in general and TEAD4 in particular are important regulators of muscle

\footnotetext{
${ }^{1}$ Institut de Génétique et de Biologie Moléculaire et Cellulaire, CNRS/INSERM/UDS, 1 Rue Laurent Fries, 67404 Illkirch Cédex, France

${ }^{*}$ Corresponding author: I Davidson, Functional Genomics and Cancer, Institut de Génétique et de Biologie Moléculaire et Cellulaire, CNRS/INSERM/UDS, 1 Rue Laurent Fries, Illkirch 67404, France. Tel: + 3338865 3445; Fax: + 3338865 3201; E-mail: irwin@igbmc.fr

Keywords: MYOD1; ER-stress; myoblast fusion; Chromatin immunoprecipitation; RNA-seq

Abbreviations: TEA, Yeast (TEC-1), Aspergillus nidulans (AbaA) and Drosophilla (scalloped); ATTS, Aspergillus nidulans (AbaA), Yeast (TEC-1), human TEF1, and Drosophilla (scalloped); DBD, DNA-binding domain; SV40, simian virus 40; CTGF, connective tissue growth factor; $\beta$-MHC, myosin, heavy polypeptide 7, cardiac muscle, beta; YAP1, Yes-associated protein 1; WWTR1, WW domain containing transcription regulator 1; ChIP-chip, chromatin immunoprecipitation-array hybridization; Flag, FLAG octapeptide tag; HA, Hemagglutinin tag; TSS, transcription start site; UPR, unfolded protein response; Cdkn1a, cyclin-dependent kinase inhibitor 1A (P21); TRP53, transformation-related protein 53; SP1, trans-acting transcription factor 1; RT-qPCR, reverse transcription-quantitative polymerase chain reaction; VGL, vestigial

Received 10.12.10; revised 11.5.11; accepted 12.5.11; Edited by B Dynlacht; published online 24.6.11
} 
development, yet few target genes have been identified. In addition, how can the contrasting roles of TEADs in proliferation and oncogenic transformation, and in cell cycle arrest and differentiation in muscle be explained?

\section{Results}

TEAD factor activity is essential for $\mathrm{C} 2 \mathrm{C} 12$ differentiation and TEAD4 plays a non-redundant role in myoblast fusion. Affymetrix gene expression data shows that undifferentiated and differentiated $\mathrm{C} 2 \mathrm{C} 12$ cells express mRNA for all members of the TEAD family (data not shown) and TEAD4 expression is strongly upregulated during differentiation (Figures $1 \mathrm{a}$ and $\mathrm{b}$ lanes 1-4). To bypass potential redundancy between the closely related TEAD proteins, we generated $\mathrm{C} 2 \mathrm{C} 12$ cell populations stably expressing a FLAG octapeptide tag (Flag-tagged)-TEAD4DBD fused to a nuclear localisation signal. The DBD, which is essentially identical in all TEADs, is not involved in coactivator interactions, and can be used as a dominant negative inhibitor of TEAD function. ${ }^{13}$ DBD expression (Figure 1c, lane 2 ) leads to diminished upregulation of TEAD4 during differentiation, loss of normal MYOG, CDKN1A induction, and delayed and diminished $\beta \mathrm{MHC}$ expression (Figure $1 \mathrm{~b}$ ). In agreement with these observations, the TEAD4-DBD almost completely inhibits differentiation, where only a few short fused myotubes are observed (Figure 2a). In contrast, empty vector-derived control cell populations differentiated similarly to uninfected $\mathrm{C} 2 \mathrm{C} 12$ cells (Figure $2 \mathrm{a}$ ). The activity of one or several TEAD factors is therefore essential for $\mathrm{C} 2 \mathrm{C} 12$ cell differentiation.

The best candidate to have an important role in muscle differentiation is TEAD4. Using lentiviral vectors, we generated cell populations expressing a scrambled shRNA control (ShSC) or two shRNAs, A and B, that knockdown TEAD4 expression (Figures $1 \mathrm{a}$ and $\mathrm{d}$, lanes 5-12). TEAD4 silencing induced shortened myotubes compared with the ShSC control (Figure 2a) that are clearly seen upon staining with antibody against $\beta \mathrm{MHC}$ (Figure 2b). ShA- and ShB-derived myotubes are shorter than controls, with mainly $2-5$ MYOD1 expressing nuclei per myotube (Figure 2d). However, these cells express $\beta \mathrm{MHC}$ indicating that they initiate differentiation, but fail to efficiently fuse as long myotubes. Labelling with TEAD4 antibody revealed incomplete knockdown with a small number of positively staining nuclei. The residual expressing cells may be able to undergo limited fusion (Figure $2 \mathrm{c}$ ).

Dexamethasone (Dex) augments myoblast fusion. ${ }^{14}$ Treatment of ShSC cells with Dex promoted formation of thickened myotubes that fused into syncytial structures (Supplementary Figure 1A) and a more rapid induction of TEAD4 expression (Supplementary Figure 1B, lanes 1-8). Treatment of ShB cells also augmented their fusion, leading to myotubes resembling those of the ShSC cells in the absence of Dex. However, whereas Dex led to a partial rescue of the TEAD4 knockdown phenotype, the ShB cells failed to show the extensive fusion seen with the control cells.

These results show that TEAD factor activity is essential for proper myoblast differentiation and that TEAD4 promotes normal myoblast fusion during differentiation. a

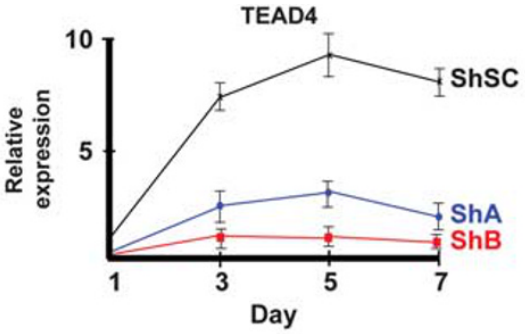

b

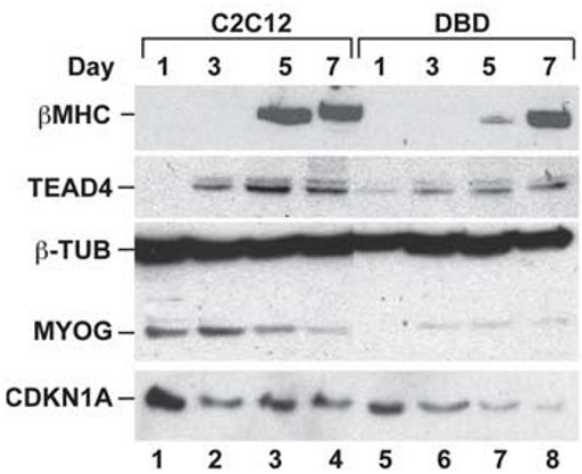

C

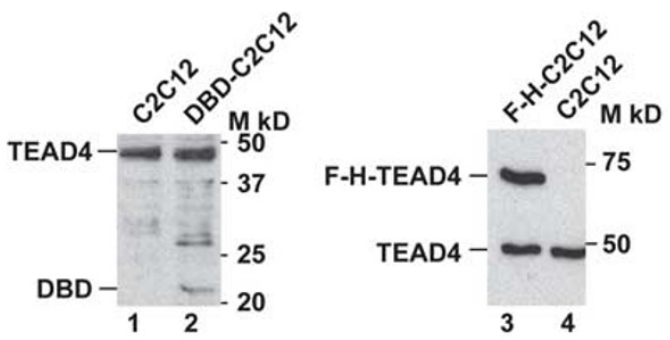

d
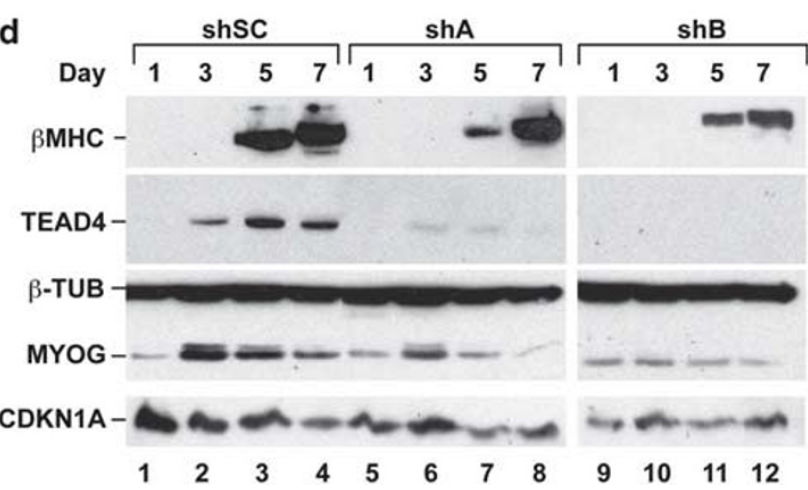

Figure 1 Expression TEAD factors in $\mathrm{C} 2 \mathrm{C} 12$ differentiation (a) RT-qPCR of TEAD4 expression during days 1-7 of $\mathrm{C} 2 \mathrm{C} 12$ cell differentiation in cells expressing the ShSC, ShA and ShB shRNAs. (b) Immunoblot analysis of the expression of the indicated proteins during differentiation of control $\mathrm{C} 2 \mathrm{C} 12$ cells and cells expressing the TEAD4-DBD. (c) Immunoblot analysis of undifferentiated C2C12 cells stably expressing the Flag-tagged TEAD4-DBD or Flag-HA-tagged full-length TEAD4. Full-length TEAD4 was detected using anti-TEAD4 antibodies, whereas the TEAD4DBD was detected with anti-Flag antibodies. (d) Immunoblot analysis of the expression of the indicated proteins during differentiation of $\mathrm{C} 2 \mathrm{C} 12$ cells expressing the indicated shRNAs

Identification of TEAD4 target genes. We established C2C12 cells stably expressing Flag-HA (hemagglutinin tag)tagged (F-H-)TEAD4 (Figure $1 \mathrm{~b}$ lanes, 3 and 4). The level of exogenous tagged TEAD4 is comparable to the endogenous 


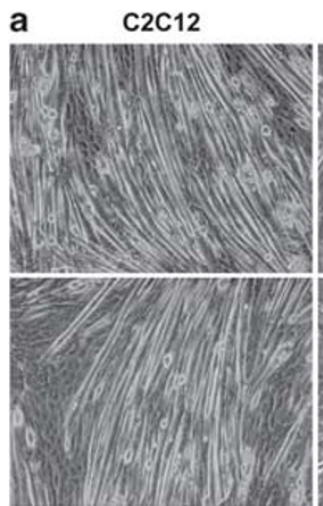

Empty vector

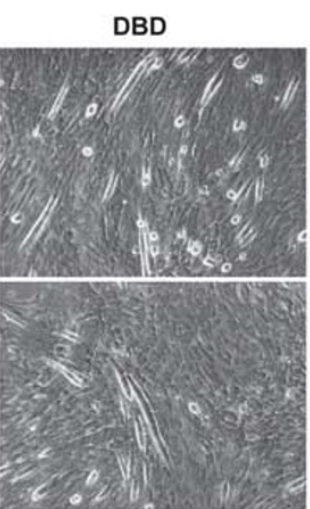

DBD

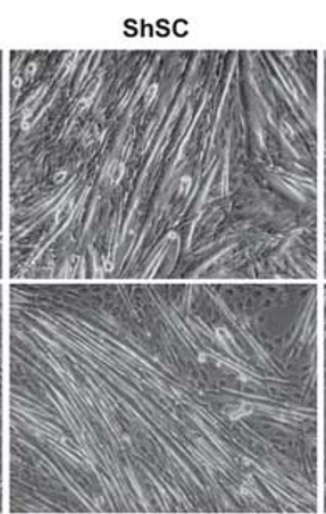

Shsc

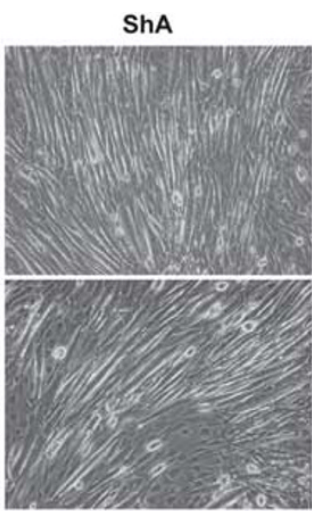

ShB b
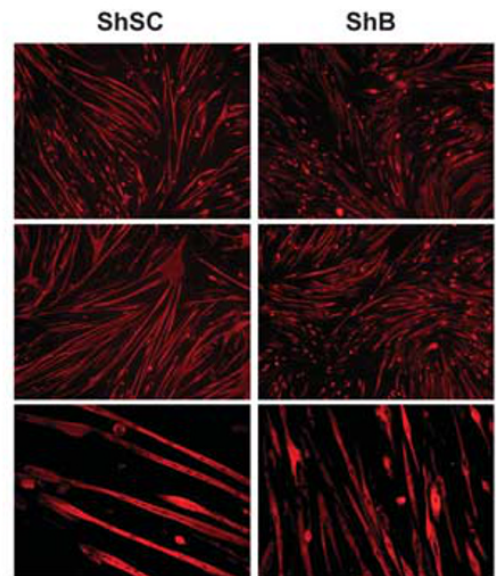

c

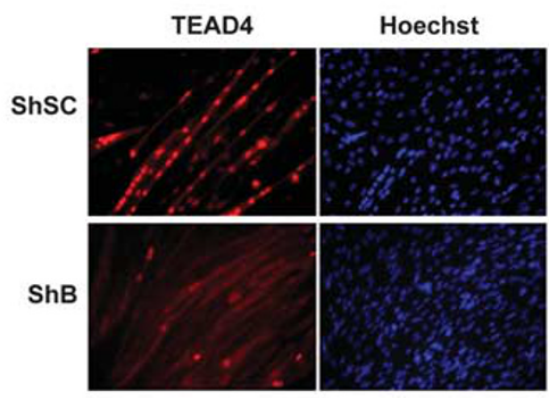

ВMHC

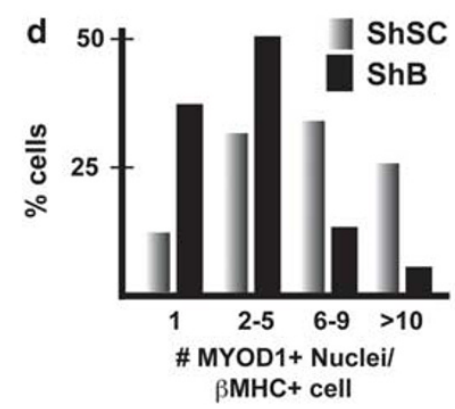

Figure 2 Differentiation of C2C12 cells expressing the TEAD4-DBD or the TEAD4 shRNAs. (a) Phase-contrast images of unselected C2C12 cells or the indicated selected $\mathrm{C} 2 \mathrm{C} 12$ cell populations at day 7 of differentiation. Magnification $\times 20$. (b) Macro-immunofluorescence of the indicated $\mathrm{C} 2 \mathrm{C} 12$ cells at day 7 of differentiation using anti- $\beta$ MHC antibody. Magnification $\times 10$ in upper two panels and $\times 20$ in lower panel. (c) Immunofluorescence of the indicated C2C12 cells stained with Hoechst or labelled with antiTEAD4 antibody. Magnification $\times 20$. (d) Day 7 differentiated ShSC or ShB-expressing cells were stained with antibodies against MYOD1 to label nuclei and anti- $\beta$ MHC to identify cells that had initiated differentiation. The results shown are the sum of two independent experiments. For the ShSC cells, 312 nuclei in 45 myotubes were counted in one experiment and 298 nuclei in 42 in the second. For ShB, 260 nuclei in 70 myotubes were counted in the first experiment and 283 nuclei in 85 myotubes in the second. The data represent the number of MYOD1 positive nuclei per $\beta$ MHC-expressing cell

tagged TEAD4 in undifferentiated cells, but is not upregulated like the endogenous protein and hence constitutes less of the TEAD4 population in differentiated cells.

We performed anti-Flag ChIP-qPCR on the F-H-TEAD4 and control untagged cells differentiated for 5 days to assess occupancy of the MCAT motif of the skeletal muscle alpha 1 actin (Acta1) gene. Clear enrichment was observed in
F-H-TEAD4 cells compared with control cells, whereas no enrichment is seen in either cell type at the control Protamine 1 (Prm1) promoter (Figure 3a).

ChIPed DNA from three independent experiments was amplified and hybridised to the Agilent extended promoter array (Materials and methods and Supplementary Text). This analysis identified 867 promoters with at least one TEAD4-occupied site (Supplementary Table 1). Taking 

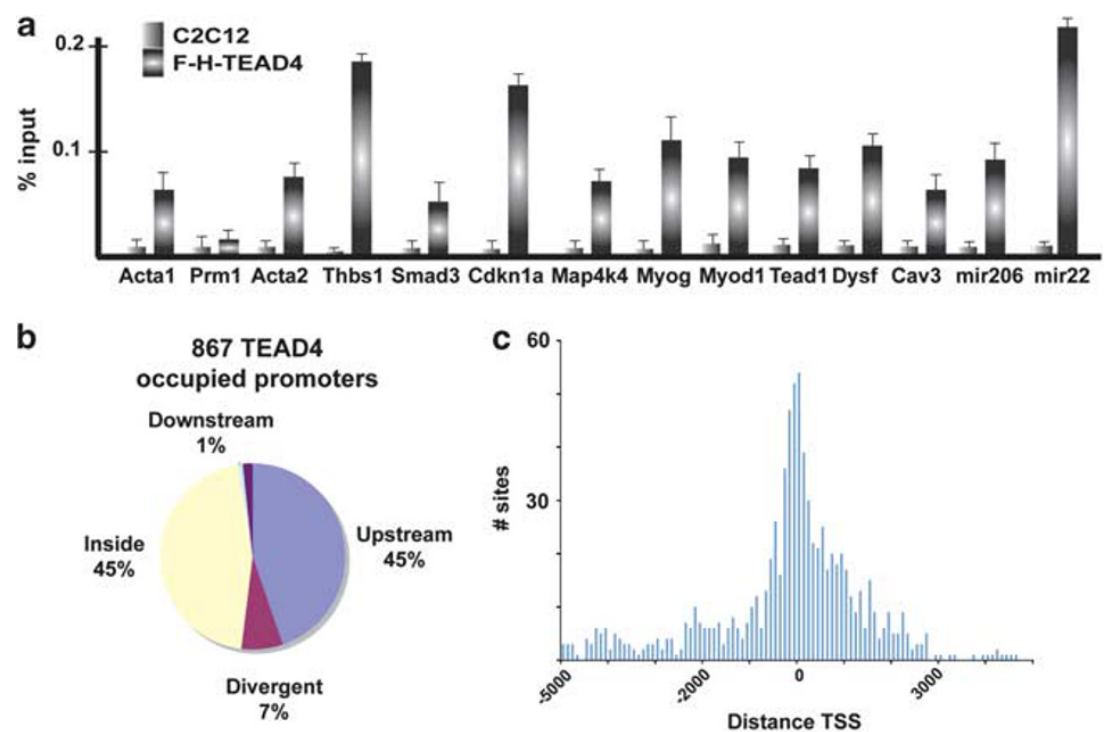

d
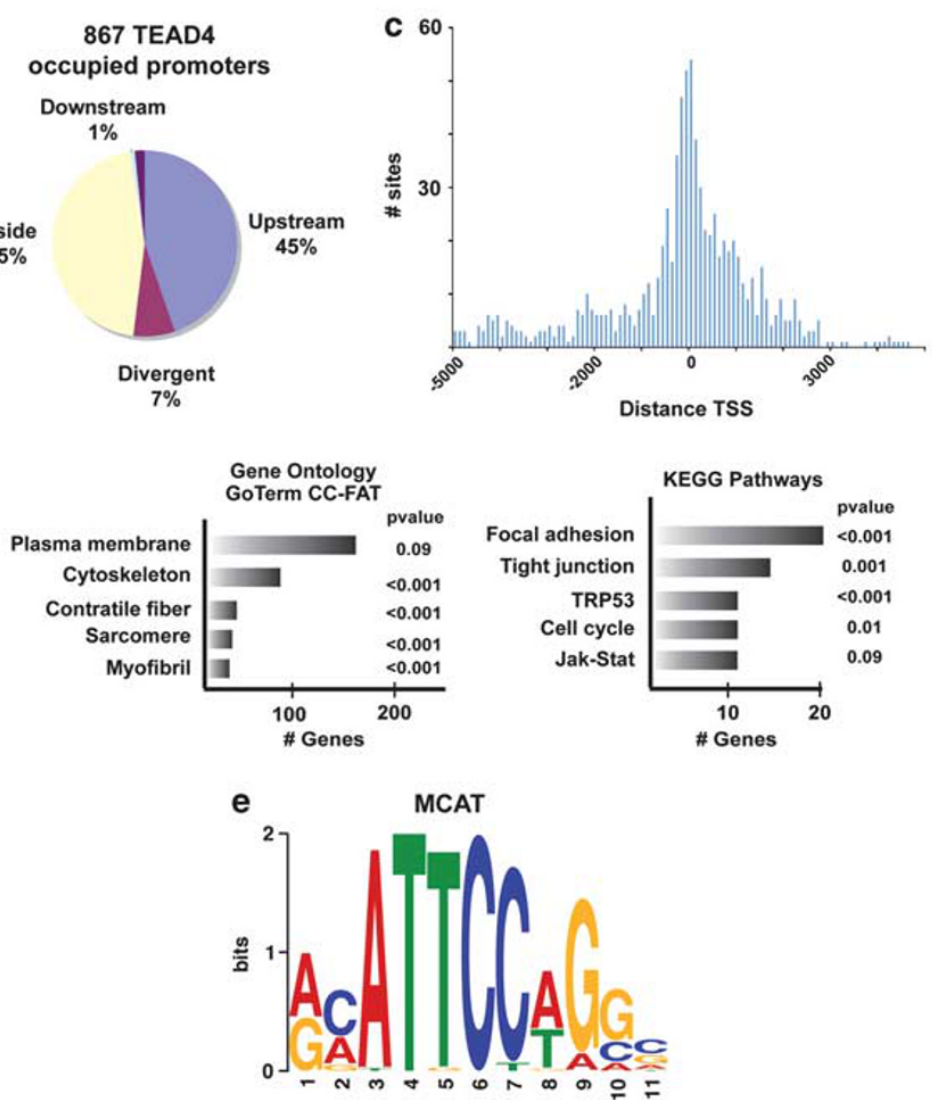

Figure 3 ChIP-chip identification of TEAD4 occupied promoters. (a) Anti-Flag ChIP-qPCR on control C2C12 cells and cells expressing Flag-HA-tagged TEAD4. QPCR was performed on amplicons from the indicated promoters and expressed as the \% input. (b) Pie chart showing the location of the TEAD4 binding sites relative to the TSS using the Agilent array annotation. (c) Location of the TEAD4-binding sites relative to the TSS. (d) DAVID (http://david.abcc.ncifcrf.gov/) ontology analysis of the TEAD4 target genes. (e) The MCAT consensus motif sequence identified by de novo Meme analysis of TEAD4 occupied sites

into account the divergent promoters, a total of 929 genes are potentially regulated by TEAD4. Bound sites were almost equally distributed between the upstream promoter and the downstream region of the transcription start site (TSS), but were enriched in the region close to the TSS (Figures $3 \mathrm{~b}$ and c). TEAD4 occupies sites at the Myog, stretch responsive muscle ankyrin repeat domain 2 (Ankrd2), Talin 1 (TIn1), Dysferlin (Dysf) Synaptogamin 8 (Syt8) and Myod1 genes (Figure 4 and Supplementary Table 3). Ontology analysis revealed potential functions for TEAD4 such as sarcomere, contractile fiber and cytoskeleton related to muscle differentiation, but also transcription regulation, cell cycle and the transformation-related protein 53 (TRP53) signalling pathway including TRP53 itself, as well as oncogenes and anti-oncogenes (Figure 3d and Supplementary Table 2).

TEAD4 also occupies sites at 17 miRNA genes including Mir-206, Mir-214 (Supplementary Table 1), a site between
Mir-1-1 and Mir-133a-2 and two sites located at the locus encoding Mir-1-2 and 133a-1 (Supplementary Figure 2). Like Mir-206, these miRNAs have important roles in C2C12 cell differentiation. ${ }^{15}$ Occupancy of several of these sites was confirmed by ChIP-qPCR (Figure 3a).

MEME (http://meme.sdsc.edu/meme4_6_1/intro.html) analysis of the TEAD4-occupied sites identified a consensus MCAT motif (Figure 4e). 475 MCAT motifs were found at 373 TEAD4-occupied sites (Supplementary Table 3). At the Myog and Myod 1 promoters (located at -5237 to -5245 and -5002 to -5011 with respect to the TSS, respectively), as well as at several of the miRNAs (Figure 4 and Supplementary Table 3) the MCAT motif was conserved among mammals, including humans, highlighting their potentially important role in regulating genes promoting myogenic differentiation. At others, the murine MCAT motif is not conserved at the same location, but MCAT motifs are present elsewhere in the human promoters (Supplementary Table 3). 


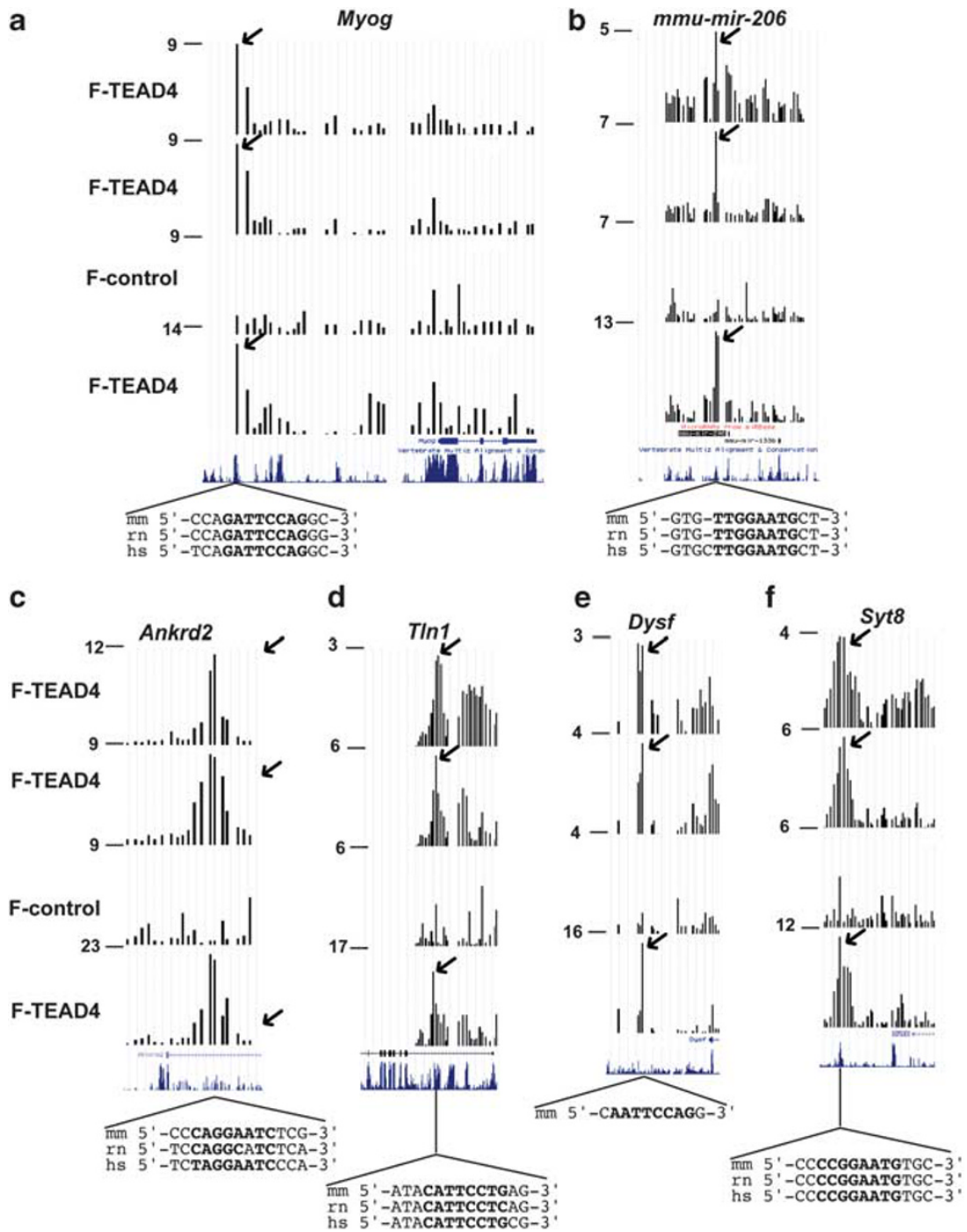

Figure 4 Representative examples of TEAD4 promoter occupancy. (a-f) Screenshots of the .Wig files in the UCSC browser of the triplicate anti-Flag ChIP-chips on the cells expressing the tagged TEAD4 (F-TEAD4) and the ChIP-chip on the un-tagged control cells (F-control) at the indicated promoters. The TEAD4-binding sites are indicated by the arrows. The values on the $Y$ axis show the normalised IP/Input ratio. The sequences of the MCAT motifs at each peak in mouse, rat and human are indicated, with the MCAT motif highlighted in bold. The locations of the TEAD4-binding sites relative to the TSS of the corresponding genes are: Myog -5237-5245, mmu-mir-206 -848-856, Ankrd2 1275-1283, TIn1 4097-4105, Dysf -7031-7039, Syt8 -4444-4452

Comparison with the MYOG ChIP-chip data where 137 MYOG-occupied promoters were identified ${ }^{10}$ showed 21 promoters co-occupied by TEAD4 (Supplementary Table 4) representing both muscle-specific genes (Acta1, and 2, Ankrd2 and Myomesin 2 (Myom2)) and generally expressed genes (Myc or Cdk5). Comparison with the MYOD1 and MEF2 ChIP-chip data from the same study identified 13 promoters co-occupied by MYOD1 and TEAD4 and 12 by TEAD4 and MEF2, again comprising muscle-specific and generally expressed genes (Supplementary Table 4). Several promoters (Acta1, Ing3 and Myog) are occupied by all three factors.

Direct regulation of $\mathrm{C2C12}$ differentiation genes by TEAD4. ChIP indicates that TEAD4 occupies a conserved MCAT motif in the Myog promoter (Figure 4a). MYOG expression is normally stimulated between 1-3 days of differentiation and persists until day 7 (Figure 1d, lanes 1-4) but is reduced in ShA cells, and almost completely repressed in ShB cells (lanes 5-12) and those expressing the DBD (Figure 1c, lanes 5-8). Reverse transcription-quantitative polymerase chain reaction (RT-qPCR) confirmed the lack of Myog activation in ShB cells (Figure 5a) showing that TEAD4 binds the Myog promoter and directly activates its expression during differentiation. In contrast, TEAD4 knockdown does not affect MYOD1 expression in the ShB cells (Supplementary Figure 3).

Activation of cyclin-dependent kinase inhibitor 1A (p21) (Cdkn1a) by MYOD1 is involved in cell-cycle arrest during myogenic differentiation. ${ }^{16,17}$ TEAD4 occupies a site in the first intron of the Cdkn1a gene (Figure 3a, and Supplementary Figure 4A) and although Cdkn1a is strongly expressed in 
a

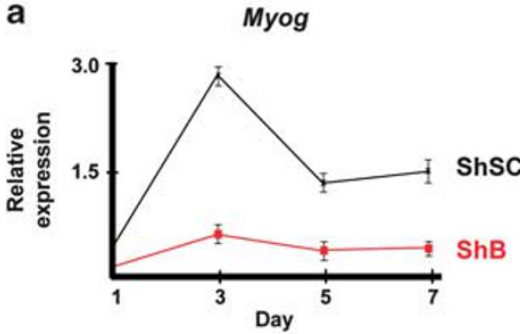

C

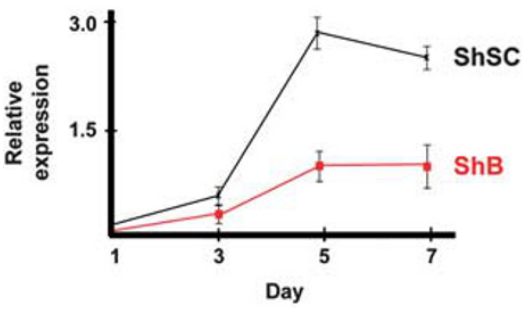

e

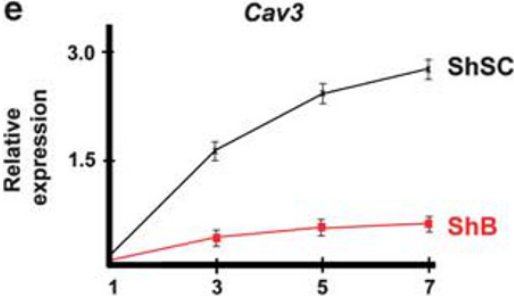

b

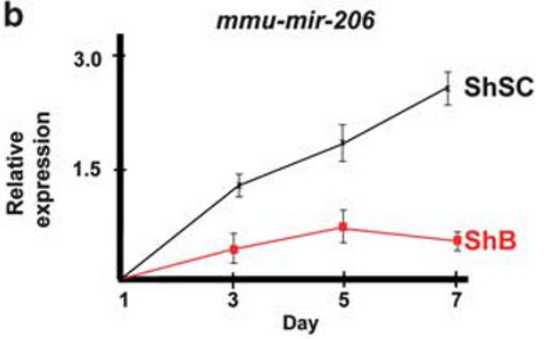

d

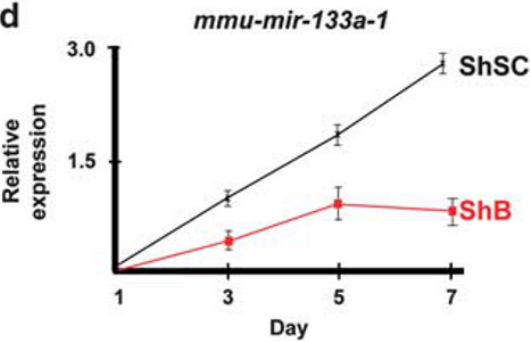

f

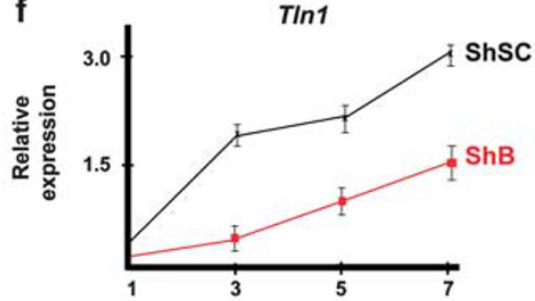

g

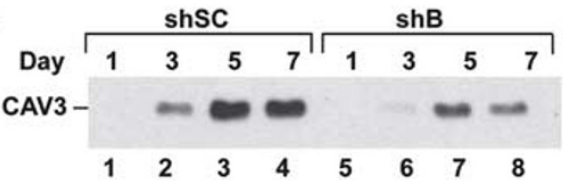

h

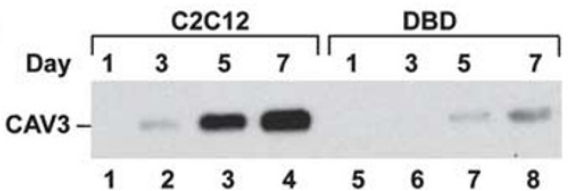

Figure 5 Changes in gene expression upon TEAD4 knockdown. (a-f) RT-qPCR quantification of the expression of the indicated genes in control ShSC-expressing C2C12 cells and cells expressing ShB from day 1 to day 7 of differentiation. Error bars show standard deviation (g and $\mathbf{h})$ Immunoblot analysis of CAV3 expression during differentiation of the indicated cell lines

control cells, its expression is diminished in the ShB or DBD cells (Figures 1c and d). Both TEAD factors and MYOD1 are therefore required for the normal and timely activation of $M y o g$ and Cdkn1a expression in $\mathrm{C} 2 \mathrm{C} 12$ differentiation.

ChIP-chip shows TEAD4 occupancy of several muscle miRNAs promoters whose expression is induced and which have essential functions in differentiation (Figure 3 and Supplementary Figure 2). RT-qPCR shows that expression of Mir-206, Mir-1-2 and Mir-133a-1 are all significantly downregulated in ShB cells (Figures 5b-d). TEAD4 is therefore required for their normal activation during differentiation.

As TEAD4 knockdown leads to shortened myotubes, we looked for direct TEAD4 target genes involved in the fusion process. TEAD4 occupies the promoters of the Dysf, TIn1, Caveolin 3, (Cav3), and Musculoskeletal, embryonic nuclear protein 1 (Mustn1) genes involved in myoblast fusion (Figures $4 \mathrm{~d}$ and e, and Supplementary Figures 4B-F). Expression of
Cav3 mRNA (Figure 5e) and protein (Figures $5 \mathrm{~g}$ and $\mathrm{h}$ and Supplementary Figure 1B) are significantly reduced upon TEAD4 knockdown or DBD expression, whereas specific, but less pronounced, effects are seen with others such as $T \ln 1$ (Figure 5f). TEAD4 therefore directly regulates CAV3 expression contributing to normal myoblast fusion.

Novel TEAD4 regulated genes. To comprehensively identify genes deregulated on TEAD4 knockdown, we performed mRNA-sequencing (mRNA-seq) on day 7 on differentiated ShSc, ShA and ShB cells. TEAD4 knockdown upregulated 518 genes and downregulated 438, most of which showed similar deregulation in ShA and ShB cells (Supplementary Table 5). Comparison of mRNA-seq and ChIP-chip identified 87 direct targets whose regulatory regions are occupied by TEAD4 (Supplementary Table 5) like the myosin light chain 2 (Myl2) gene whose expression is 

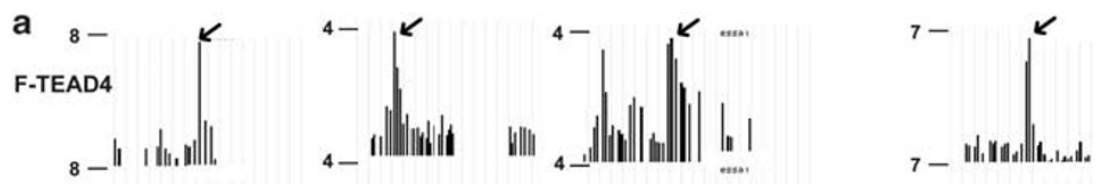

F-control

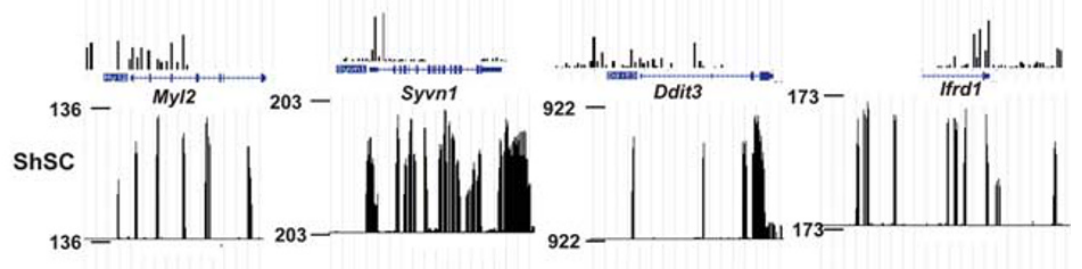

ShB
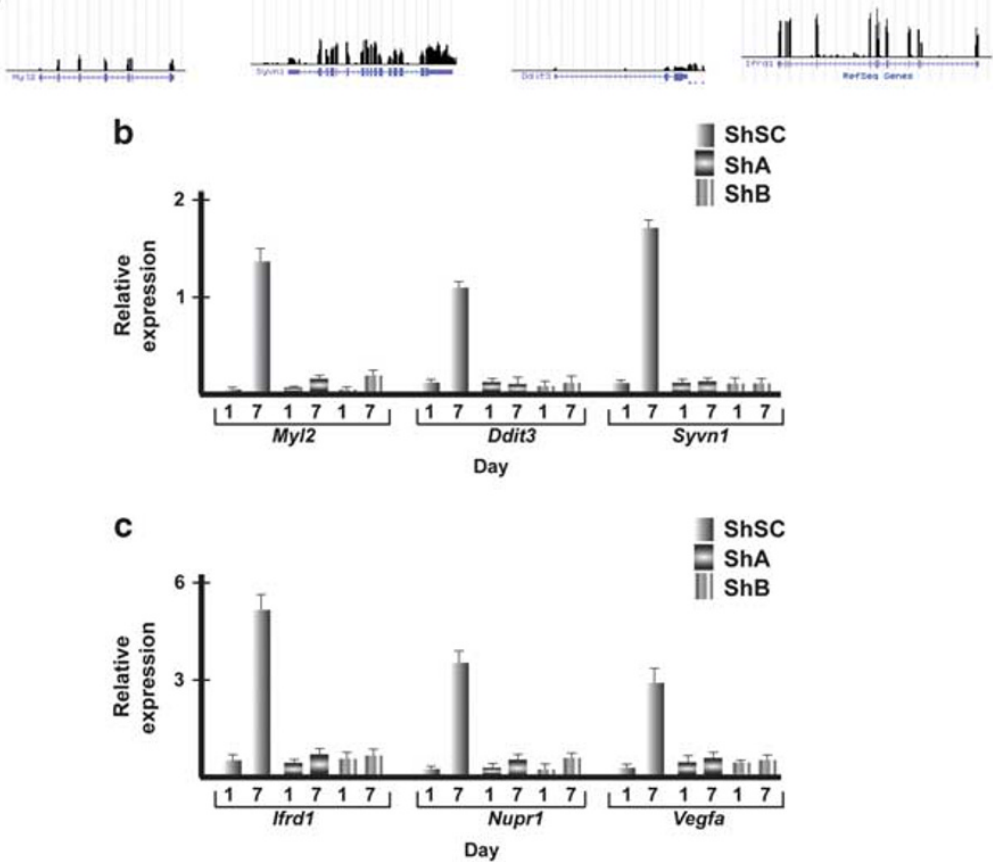

Figure 6 TEAD4 regulation genes required for myoblast fusion. (a) Screenshots of the Wig files in the UCSC browser of the anti-Flag ChIP-chip and the mRNA-seq at the indicated promoters. (b and $\mathbf{c}$ ) RT-qPCR quantification of the indicated genes in the indicated cell lines. The locations of the TEAD4-binding sites relative to the TSS of the corresponding genes are: Myl2, 2121-2129, Syvn1 96-104, Ddit3 -248-256, Ifrd1-284-292

strongly reduced in ShA and ShB knockdown cells (Figures $6 a$ and $b)$.

Among the downregulated genes, are those encoding the endoplasmic reticulum (ER) chaperones and ER-associated degradation proteins that make up the unfolded protein response (UPR). Expression of Hspa5 (mBIP), Syvn1 (HDR), Ddit3 (CHOP), and its heterodimerisation partner Cebpb, Sel11, Dnajc3, Gadd45a, Ppp1r15a (GADD34) and Chac1 are all strongly downregulated (Figure $6 a$ and Supplementary Table 5). RT-qPCR indicates that Ddit3 and Syvn1 are strongly induced upon normal differentiation, but not in ShA and ShB cells (Figure 6b). These genes are direct targets with TEAD4 occupied sites within their regulatory regions (Figure 6a).

ER-stress and the UPR are reported to be activated during myogenic and keratinocyte differentiation. ${ }^{18-20}$ ER stress activates IRE1 that splices the Xbp1 mRNA generating an isoform encoding the XBP1 transcription factor. ${ }^{21,22}$
Quantification of the mRNA-seq reads that cover the splice junctions in exon 4 of the Xbp1 mRNA in the ShSC, ShA and ShB cells showed that around half the Xbp1 mRNA was spliced in the ShSC cells, but not in TEAD4 knockdown cells (Supplementary Figure 5A). RT-PCR generating products that distinguish the spliced and unspliced forms confirmed that the spliced form was present only at day 7 in the ShSC cells, but not in TEAD4 knockdown cells (Supplementary Figure 5B). ER-stress induced UPR is mediated by transcription factors ATF6 and ATF ${ }^{23}$ that are expressed in control cells, but strongly downregulated in ShA and ShB cells, although they do not appear to be direct TEAD4 targets. Other UPR/ER stress response genes (Herpud1, Bbc3 (PUMA), Hsp90b1, Pdia4; Atp2a3, Ern1, Car6, Xbp1 and Sdf2/1) are also indirectly downregulated, perhaps due to the lack of DDIT3/CEBPB transcription factor induction. ER stress and the UPR are thus activated during normal $\mathrm{C}_{2} \mathrm{C} 12$ differentiation, but not in incompletely differentiated TEAD4 knockdown cells. 

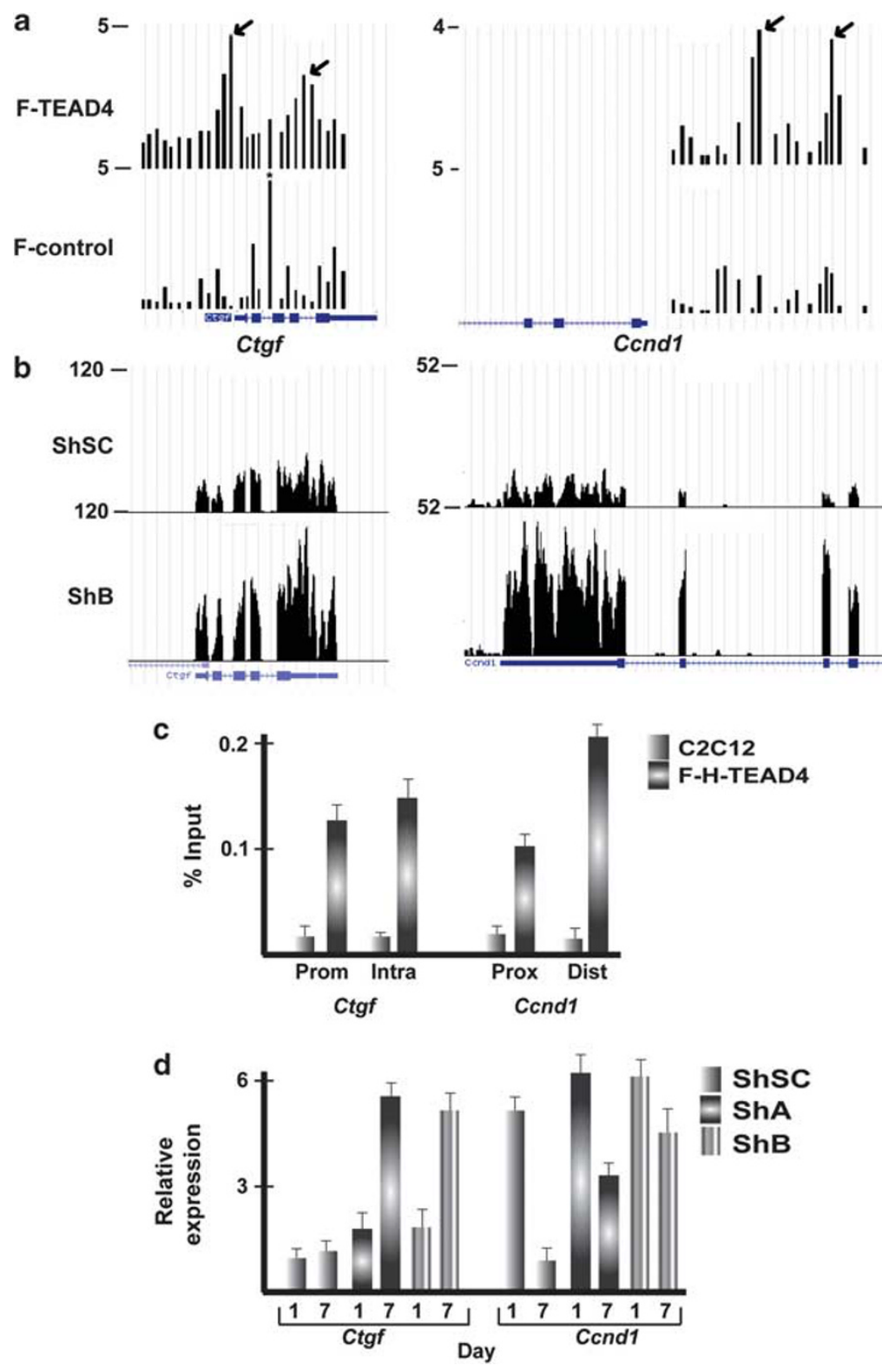

Figure 7 TEAD4 represses CTGF and CCND1 expression. (a and $\mathbf{b})$ Screenshots of the .Wig files in the UCSC browser of the anti-Flag ChIP-chip and the mRNA-seq at the Ctgf and Ccnd1 promoters. (c) Flag-ChIP-qPCR showing TEAD4 occupancy at the two sites in the Ctgfand Ccnd1 promoters. (d) RT-qPCR quantification of the indicated genes in the indicated cell lines. The locations of the TEAD4-binding sites relative to the TSS of the corresponding genes are: Ctgf downstream 1674-1682, Ccnd1 distal $-3737-3745$, Ccnd1 proximal -2075-2083

Our data also show that TEAD4 directly regulates expression of Nupr1 (Figure 6c), encoding p8, a small HMG-related chromatin protein that is essential for cell cycle exit and $M y o g$ activation in differentiating $\mathrm{C} 2 \mathrm{C} 12$ cells, ${ }^{24}$ and interferonrelated developmental regulator 1 (Ifrd1; Figures $6 \mathrm{a}$ and b), a transcriptional corepressor interacting with $\mathrm{mSin} 3 \mathrm{~B}$ and histone deacetylase 1 to repress transcription. ${ }^{25}$ Mice lacking Ifrd1 show progressive loss of muscle fibers and severely reduced muscle regeneration. ${ }^{26}$ Myogenic precursor cells from mutant animals show severely reduced differentiation and fusion in vitro. Reduced expression of the Nupr1 and Ifrd1 genes upon TEAD4 knockdown may contribute to the observed differentiation defects and may be critical target genes mediating TEAD4 function. TEAD4 additionally activates VEGFA expression (Figure 6c) to promote myogenic differentiation.

TEAD4 represses CTGF expression to promote myogenic differentiation. TEAD factors interact with YAP1 to promote proliferation through activation of $\operatorname{Ctgf}^{12}$ or Ccnd1. ${ }^{27}$ ChIP-chip and ChIP-qPCR shows TEAD4 occupancy of two sites, one in the Cgtf promoter and one intragenic, and two sites in the proximal and distal regions of the Ccnd1 promoter (Figures $7 \mathrm{a}$ and $\mathrm{c}$ and Supplementary Table 3). Ccnd1 expression was downregulated during differentiation of ShSC cells, but not in ShA and ShB cells 
(Figures $7 \mathrm{~b}$ and d). Similarly, Ctgf expression does not significantly change in differentiating ShSC cells, but is upregulated in ShA and ShB cells. Thus, whereas TEADs activate Ctgf expression to promote proliferation in nonmuscle cells, TEAD4 represses its expression in differentiating $\mathrm{C} 2 \mathrm{C} 12$ cells.

\section{Differential TEAD4 target site occupancy during differentiation. TEAD4 regulates both constitutively expressed and differentiation-induced genes. ChIP-qPCR shows that TEAD4 occupies the distal site in the Cond1 promoter in undifferentiated cells, and occupancy does not strongly vary during days 1,3 and 5 of differentiation (Figure 8a). A similar profile is seen at the Ifrd1 promoter, despite the fact that it is induced only during differentiation (Figure 8b). The Myog promoter shows significant occupancy in undifferentiated cells that further increases during differentiation (Figure 8c). In contrast, TEAD4 occupancy of Cav3, and Nupr1, as well as the UPR genes Ddit3, Synv1, Hspa5 and Cebpb, is low in undifferentiated cells and strongly increases only between days 3 and 5 of differentiation (Figures $8 d-i$ ). \\ Together these results show differential occupancy of TEAD4 target sites between those that are constitutively occupied like Ccnd1, and those of differentiation-induced genes that are fully occupied only at later stages of differentiation.}

\section{Discussion}

TEAD factors and MYOD1 are required for proper initiation of myoblast differentiation. Myoblast differentiation requires cell-cycle exit, expression of muscle structural proteins forming the contractile fiber, specialised cell junctions, myoblast fusion and expression of the neuromuscular junction genes. This process is driven by MYOD1 that activates MYOG and MEF2, which, then, together regulate downstream genes involved in the above processes. ${ }^{10,28}$ TEAD4 was considered a downstream target of MYOD1 and MYOG required to regulate muscle structural genes. Our results show that TEAD factors indeed regulate downstream structural genes, but also are essential at the initial steps of differentiation.

TEAD4 occupies a conserved MCAT motif in the Myog promoter and Myog activation is strongly reduced in the TEAD-DBD or TEAD4 knockdown cells. As diminished Myog activation cannot be ascribed to loss of MYOD1, both MYOD1 and TEAD4 must be required for normal Myog activation. Both TEAD4 and MYOD1 occupy the Myog promoter in undifferentiated cells and their occupancy increases during differentiation. ${ }^{28}$ Pre-bound MYOD1 and TEAD4 do not activate Myog transcription in undifferentiated cells as activation requires MEF2- and p38 kinase-dependent recruitment of the histone methyltransferases Ash2L and MLL to promote H3K4me3, and Six4 recruitment of the UTX histone demethylase to remove repressive marks. ${ }^{29}$ Similarly, Cdkn1a is activated in the initial stage of normal differentiation, whereas its expression is diminished by TEAD4 knockdown. Both the TEAD and MYOD1 factors are therefore required for normal activation of Myog and Cdkn1a and proper initiation of myogenic differentiation.
MYOD1 and MYOG bind to the Tead4 promoter, ${ }^{10}$ whereas we show that TEAD4 binds to the Myog promoter. Activation of Myog expression and differentiation therefore requires a positive feedback loop between the TEAD factors, MYOD1 and MYOG. NUPR1 may act at the Myog promoter to facilitate recruitment of MYOD1 and p300 to promote Myog expression. ${ }^{24}$ Nupr1 expression is directly regulated by TEAD4 suggesting that TEAD4 acts also indirectly on Myog expression through regulation of Nupr1.

We describe the role of TEAD4 during differentiation, but many TEAD4-occupied genes are not induced during differentiation. As we did not observe any changes in the proliferation of the TEAD4 knockdown myoblasts, the role of TEAD4 in C2C12 cells therefore appears to be mainly related to differentiation.

Regulation of UPR genes during myogenic differentiation. TEAD4 silencing shows a weaker phenotypic effect than blocking all TEAD factors with the DBD. TEAD4 is therefore a major actor in differentiation, but shows partial redundancy with other TEADs. TEAD4 silencing leads to shortened myotubes that may, in part, be accounted for by downregulation of CAV3, but also of Nupr1 and Ifrd1 and/or more generally incomplete differentiation. Nevertheless, expression of many direct target muscle structural genes, as well as those involved in fusion such as Dysferlin, is either mildly or unaffected by TEAD4 knockdown, suggesting extensive functional redundancy between the TEAD factors at these promoters.

In contrast, a specific set of direct target genes are strongly downregulated suggesting that TEAD4 has essential nonredundant functions at their promoters, among which are essential UPR components like Ddit3 and Cebpb, whose promoters are normally progressively occupied by TEAD4. In contrast, their activation is lost in TEAD4 knockdown cells along with their targets like $B b c 3$ and Car6. $^{30} \mathrm{Xbp} 1$ is regulated by $M Y O D 1$ and $M Y O G^{20}$ and is also indirectly downregulated by TEAD4 silencing.

Our study shows that $\mathrm{C} 2 \mathrm{C} 12$ differentiation is normally accompanied by ER stress, splicing of the Xbp1 mRNA, expression of the ATF4 and ATF6 mediators of ER stress and activation of the UPR genes. In contrast, all of these events are reduced upon TEAD4 silencing. The lack of UPR gene activation in TEAD4 knockdown cells may reflect a TEAD4 requirement to activate their expression as suggested by its occupancy of the promoters of many of these genes, but also the lack of a potent ER-stress response due to the generally altered differentiation of the shTEAD4 cells. Future experiments will determine whether TEAD4 is directly required for activation of the UPR genes either as a component of the ER stress response and/or in a differentiation-associated pathway.

Cardiac hypertrophy is an adaptive response of the heart to increased workload and injury that occurs in a number of physio-pathological conditions. In this study, we show that TEAD4 directly regulates expression of NUPR1 that is required for endothelin- and $\alpha$-adrenergic agonist-induced cardiomyocyte hypertrophy. ${ }^{31}$ Moreover, NUPR1 negatively regulates cardiomyocyte autophagy. Dysfunctional autophagy has been linked to cardiomyopathies and hypertrophy. ${ }^{32}$ 


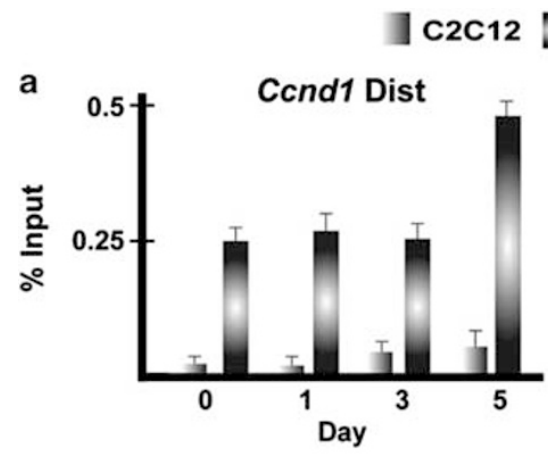

\section{F-H-TEAD4}
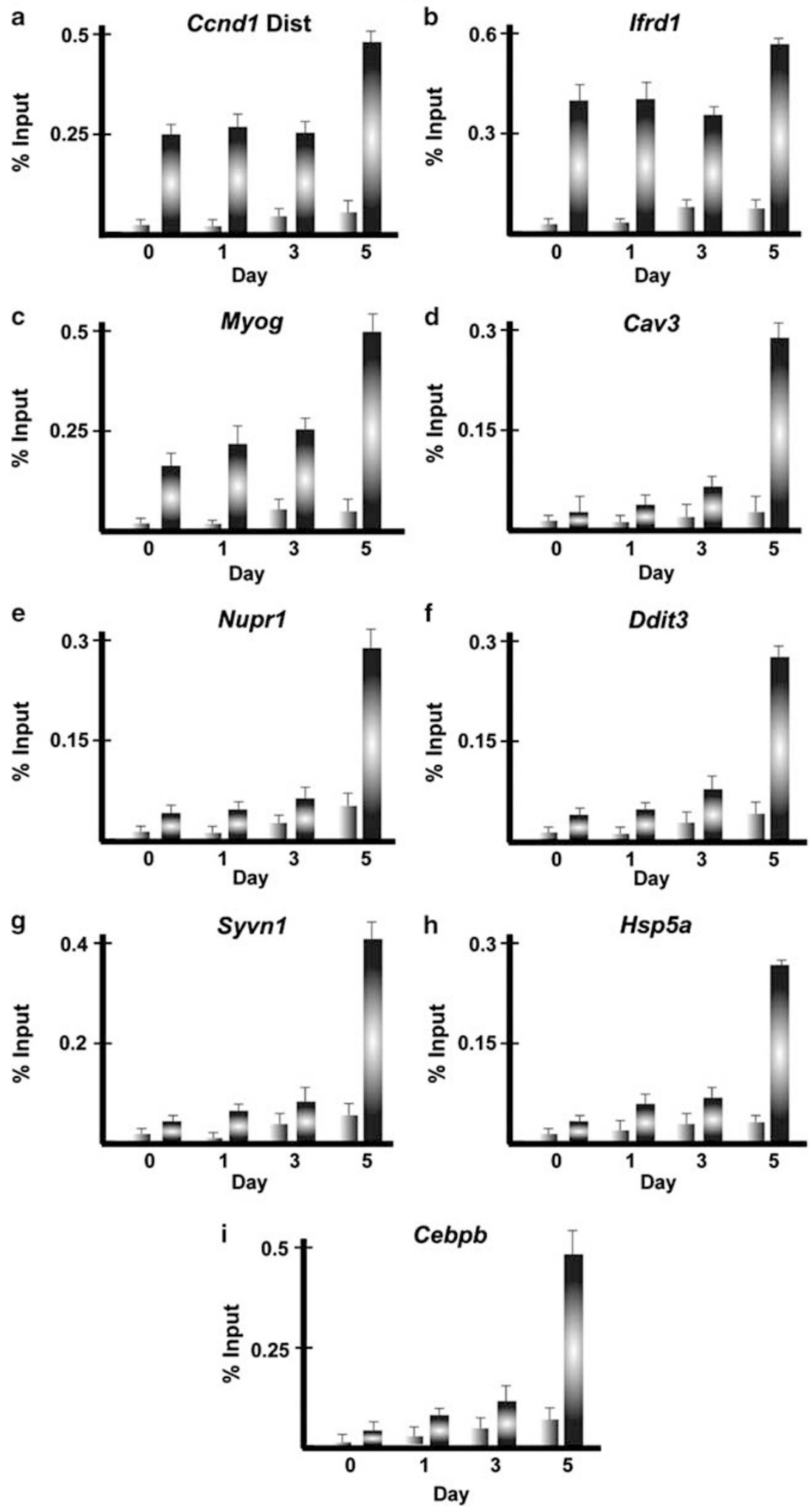

Figure 8 Differential TEAD4 target site occupancy during differentiation. (a-i) Anti-Flag ChIP-qPCR on control C2C12 cells and cells expressing Flag-HA-tagged TEAD4 in the undifferentiated state (day 0) and at days 1, 3 and 5 of differentiation. QPCR was performed on amplicons spanning the TEAD4 occupied M-CAT motifs from the indicated promoters and expressed as the \% input. The locations of the TEAD4-binding sites relative to the TSS of the corresponding genes are as described above and Cav3 -54-62, Nupr1-2813-2821, Hspa5 346-354, Cebpb -3433-3441 
It is therefore possible that TEAD4 regulation of NUPR1 in cardiomyocytes may modulate cardiac hypertrophy.

Although our observations demonstrate an important role for the TEAD factors in myoblast differentiation in vitro, this function has not been clearly demonstrated in vivo where post-implantation inactivation of TEAD4 gives rise to viable adult animals with no obvious phenotype. ${ }^{11,33}$ This may reflect compensation by other TEAD factors during development, which does not occur in vitro when an accute loss of TEAD4 expression is induced by shRNA silencing. TEAD factors clearly have redundant functions in vivo, as while TEAD2 knockout mice have only a minor phenotype, TEAD1/TEAD2 double-knockout mice have pleiotropic abnormalities that are much more severe than the loss of TEAD1 or TEAD2 alone. ${ }^{8,34}$

Cell-specific regulation of proliferation genes by TEAD
factors. In non-muscle cells, TEAD factors promote proliferation via Ctgf or Ccnd1. In C2C12 cells, TEAD4 occupies the Ctgf promoter, and in contrast to non-muscle cells, represses its expression during differentiation. This repression is critical for differentiation as CTGF inhibits C2C12 differentiation and can even induce de-differentiation. ${ }^{35}$ In addition, TEAD factors regulate $\mathrm{C} 2 \mathrm{C} 12$ cell-cycle exit through activation of Cdkn1a. Differential regulation of CTGF and CDKN1A by TEAD factors in muscle and in other cell types thus appears to have a pivotal role in the transition from proliferation to cell-cycle arrest.

This differential regulation may be due to use of different cofactors in muscle and non-muscle cells. In $\mathrm{C} 2 \mathrm{C} 12$ cells, YAP1 is phosphorylated and exported from the nucleus during differentiation. ${ }^{36}$ Expression of a non-phosphorable YAP1 mutant that remains nuclear inhibits differentiation. Nevertheless, stable expression of the TEAD4-DBD that represses differentiation has no significant effect on $\mathrm{C} 2 \mathrm{C} 12$ myoblast proliferation. YAP1 may therefore not act via TEAD factors to stimulate proliferation of $\mathrm{C} 2 \mathrm{C} 12$ cells. In contrast, the vestigial (VGL) family of proteins act as TEAD cofactors in muscle cells. ${ }^{37}$ Exchange of YAP1 for VGL cofactors may, therefore, be an essential event in muscle differentiation and account for the differential regulation of growth promoting genes.

\begin{abstract}
Materials and Methods
C2C12 cell culture and differentiation. $\mathrm{C} 2 \mathrm{C} 12$ cells were cultured and differentiated under standard conditions. Differentiation was induced by switching to medium with $2 \%$ horse serum (Gibco, Invitrogen, Carlsbad, CA, USA). Day 1 is taken as $24 \mathrm{~h}$ after switching to differentiation medium. C2C12 cell lines expressing Flag-HAtagged TEAD4 and the Flag-tagged TEAD4-DBD were generated by infection with the corresponding pBABE retroviruses, and infected cell populations were selected with continuous presence of puromycin, as described ${ }^{38}$ Cell populations expressing the shRNAs were generated by infection with the appropriate pLKO. 1 lentiviral vectors and selection with continuous presence of puromycin. The TEAD4 shRNA sequences are, shA (5'-CCGGCCGCCAAATCTATGACAAGTTCTCGAGAACTTGTCATAGATTT GGCGGTTTTG-3'), and shB (5'-CCGGGCTGAAACACTTACCCGAGAACTC GAGTTCTCGGGTAAGTGTTTCAGCTITTG-3') and were ordered from SigmaAldrich (St. Louis, MO, USA). Control shRNA, pLKO.1-scramble shRNA (1864) was from Addgene (Cambridge, MA, USA).
\end{abstract}

Immunoblots and immunofluorescence. Total cell extracts were prepared by the freeze thaw technique. Immunoblots were performed by standard techniques. The following antibodies were used: TEAD4 (M01) from Abnova (Taipei City, Taiwan), $\beta$-MHC (MY-32) from Sigma-Aldrich, MYOG (F5D),
CDKN1A (C-19) and MYOD1(C-20) from Santa-Cruz Biotechnology (Santa Cruz, CA, USA), $\beta$-TUB (AB21057) from Abcam (Cambridge, UK), CAV3 (mouse monoclonal) from BD Transduction Laboratories (San Jose, CA, USA), DYSF (mAb NCL-Hamlet-2) from Novocastra (Rungis, France). Immunofluorescence was performed by standard techniques. Briefly, cells were rinced once in PBS and fixed in $4 \%$ paraformaldehyde. Slides were blocked with $3 \%$ serum in PBS with $0.1 \%$ Triton-X100. Primary antibodies were incubated overnight at $4{ }^{\circ} \mathrm{C}$ and then washed three times with PBS $0.1 \%$ Tween-20. Slides were then incubated with fluorescentlabelled secondary antibodies, and after washing visualised by fluorescence microscopy. DNA was counterstained with Hoechst.

Chromatin immunoprecipitation. ChIP experiments were performed according to standard protocols and are described in more detail in the Supplementary Material. All ChIP was performed in triplicate and analysed by triplicate qPCR. For ChIP-chip, the total input chromatin and ChIPed material were hybridised to the extended promoter array from Agilent (Santa Clara, CA, USA) covering $-5 \mathrm{~kb}$ to $+2 \mathrm{~kb}$ regions of around 17000 cellular promoters, as previously described. ${ }^{38}$ Data were analysed with ChIP Analytics from Agilent, further details are described in the Supplementary Material. Flag ChIP was performed with AntiFlag M2 Affinity Gel (Sigma-Aldrich, A2220). Real-time PCR were performed on Roche Lightcycler using Roche SYBR Green mix (Roche, Basel, Switzerland). Primer sequences are shown in the Supplementary Table 6.

Bioinformatics analysis. The 500 nucleotides under the highest scoring oligonucleotide at each TEAD4 binding site were analysed using the MEME programme (http://meme.sdsc.edu/meme4_6_1/intro.html). Comparisons with the previously described ChIP-chips from Blais et al., ${ }^{10}$ were performed with Excel using their Supplementary Tables. The presence of MCAT motif in human promoters was perfomed on the region from $-5 \mathrm{~kb}$ to $+2 \mathrm{~kb}$ using a custom JAVA API application and allowing one mismatch compared with the consensus sequence outside the $5^{\prime}-$ TTCC'$^{\prime}-3^{\prime}$ core that was considered as invariable.

RNA extraction and quantitative real-time RT-PCR. Total RNA was extracted using an RNeasy kit from Qiagen (Valencia, CA, USA) according to the manufacturer's instructions. $1 \mu \mathrm{g}$ of RNA was reverse transcribed using AMV retrotranscriptase (Roche) using hexanucleotides. The final product was diluted 200 times and $5 \mu \mathrm{l}$ were mixed with forward and reverse primers listed in Supplementary Table 3 ( $300 \mathrm{nM}$ of each primer at final concentration) and $7.5 \mu \mathrm{l}$ of SYBR Green master mix in total volume of $15 \mu \mathrm{l}$. The real-time PCR reaction was performed using the LightCycler 1.5 system (Roche). Each cDNA sample was tested in triplicate. For quantification of gene expression changes, the $\delta \mathrm{Ct}$ method was used to calculate relative fold changes normalised against $\beta$-actin expression. Oligonucleotide primer sequences are listed in Supplementary Table 6.

MicroRNAs were extracted using an RNeasy mini kit (Qiagen) according to the manufacturer's instructions. $1 \mu \mathrm{g}$ of RNA was reverse transcribed using miScript reverse transcription kit (218061) from Qiagen. The real-time PCR was performed using miScript SYBR Green PCR Kit (218073) from Qiagen. Reverse transcription product was diluted 200 times and $5 \mu \mathrm{l}$ were mixed with $1.5 \mu \mathrm{l}$ universal primer (provided with miScript SYBR Green PCR Kit), $1.5 \mu$ l microRNA specific primer from Qiagen, $7.5 \mu \mathrm{l}$ miScript SYBR Green PCR Kit. For quantification of gene expression changes, the $\delta \mathrm{Ct}$ method was used to calculate relative fold changes normalised against small RNA U6 expression. The following primers were used: MiR-1-2 (MS00011004), miR-133a-1 (MS00007294) from Qiagen. For miR-206 and small RNA U6 the following primers were used: miR-206 (5'-TGGAATGTAAGGAAG TGTGTGG-3') and small RNA U6 (5'-CGCAAGGATGACACGCAAATTCGT-3').

mRNA-seq. The mRNA-seq libraries were prepared following the Illumina protocol with some modifications. Briefly, mRNA was purified from total RNA using oligo-dT magnetic beads and fragmented using divalent cations at $95^{\circ} \mathrm{C}$ for $5 \mathrm{~min}$. The cleaved mRNA fragments were reverse transcribed to CDNA using random primers. This was followed by second strand cDNA synthesis using Polymerase I and RNase $H$. The double strand cDNA fragments were blunted, phosphorylated and ligated to single-end adapter dimers followed by PCR amplification ( $30 \mathrm{~s}$ at $98^{\circ} \mathrm{C}$; $\left(10 \mathrm{~s}\right.$ at $98^{\circ} \mathrm{C}, 30 \mathrm{~s}$ at $65^{\circ} \mathrm{C}, 30 \mathrm{~s}$ at $\left.72^{\circ} \mathrm{C}\right) \times 13$ cycles; 5 min at $\left.72^{\circ} \mathrm{C}\right)$. After PCR amplification, surplus PCR primers and dimer adapters were removed by purification using AMPure beads (Agencourt Biosciences Corporation, Beverly, MA, USA). Size selection was performed by electrophoresis on a $2 \%$ agarose gel and DNA fragments in the range of $\sim 250-350 \mathrm{bp}$ were excised and purified using QIAquick Gel Extraction Kit (Qiagen). DNA libraries were checked for quality and 
quantified using 2100 Bioanalyzer (Agilent). The libraries were loaded in the flowcell at $6 \mathrm{pM}$ concentration and clusters generated and sequenced on the Illumina Genome Analyzer Ilx as single-end 72 base reads. Image analysis and base calling were performed using the Illumina Pipeline version 1.6 and sequence reads mapped to reference genome mm9/NCBI37 using Tophat. ${ }^{39}$ Quantification of gene expression was done using Cufflinks ${ }^{39}$ and annotations from Ensembl release 57. For each transcript the resulting FPKM were converted into raw read counts and these counts were added for each gene locus. Data normalization was performed with the method proposed by Anders et al. ${ }^{40}$ and implemented in the DESeq Bioconductor package.

\section{Conflict of Interest}

The authors declare no conflict of interest.

Acknowledgements. We thank $\mathrm{K}$ Hnia and $\mathrm{J}$ Laporte for the gift of antibodies and advice. This work was supported by grants from the CNRS, the INSERM, the Universite de Strasbourg, the Association pour la Recherche contre le Cancer, the Ligue Nationale contre le Cancer, the INCA and the AFM. ID is an 'équipe labellisée' of the Ligue Nationale contre le Cancer. AB was supported by a followship from the AFM. The mRNA-seq data discussed in this publication have been deposited in NCBl's Gene Expression Omnibus and are accessible through GEO Series accession number GSE27845 (http://www.ncbi.nlm.nih.gov/geo/query/acc.cgi? acc $=$ GSE27845). The ChIP-chip data are are accessible through GEO Series accession number GSE29208.

1. Jacquemin P, Hwang JJ, Martial JA, Dolle P, Davidson I. A novel family of developmentally regulated mammalian transcription factors containing the TEA/ATTS DNA binding domain J Biol Chem 1996; 271: 21775-21785.

2. Yoshida T. MCAT elements and the TEF-1 family of transcription factors in muscle development and disease. Arterioscler Thromb Vasc Biol 2008; 28: 8-17.

3. Burglin TR. The TEA domain: a novel, highly conserved DNA-binding motif [letter]. Cell 1991; 66: 11-12.

4. Andrianopoulos A, Timberlake WE. ATTS, a new and conserved DNA binding domain [letter]. Plant Cell 1991; 3: 747-748.

5. Anbanandam A, Albarado DC, Nguyen CT, Halder G, Gao X, Veeraraghavan S. Insights into transcription enhancer factor 1 (TEF-1) activity from the solution structure of the TEA domain. Proc Natl Acad Sci USA 2006; 103: 17225-17230.

6. Mar JH, Ordahl CP. A conserved CATTCCT motif is required for skeletal muscle-specific activity of the cardiac troponin T gene promoter. Proc Natl Acad Sci USA 1988; 85 6404-6408

7. Chen Z, Friedrich GA, Soriano P. Transcriptional enhancer factor 1 disruption by retroviral gene trap leads to heart defects and embryonic lethality in mice. Genes Dev 1994; 8: 2293-2301

8. Chen $\mathrm{HH}$, Baty CJ, Maeda T, Brooks S, Baker LC, Ueyama T et al. Transcription enhancer factor-1-related factor-transgenic mice develop cardiac conduction defects associated with altered connexin phosphorylation. Circulation 2004; 110: 2980-2987.

9. Maeda T, Mazzulli JR, Farrance IK, Stewart AF. Mouse DTEF-1 (ETFR-1, TEF-5) is transcriptional activator in alpha 1-adrenergic agonist-stimulated cardiac myocytes. J Biol Chem 2002; 277: 24346-24352.

10. Blais A, Tsikitis M, Acosta-Alvear D, Sharan R, Kluger Y, Dynlacht BD. An initial blueprint for myogenic differentiation. Genes Dev 2005; 19: 553-569

11. Yagi R, Kohn MJ, Karavanova I, Kaneko KJ, Vullhorst D, DePamphilis ML et al. Transcription factor TEAD4 specifies the trophectoderm lineage at the beginning of mammalian development. Development 2007; 134: 3827-3836.

12. Zhao B, Li L, Lei Q, Guan KL. The Hippo-YAP pathway in organ size control and tumorigenesis: an updated version. Genes Dev 2010; 24: 862-874.

13. Hwang JJ, Chambon P, Davidson I. Characterization of the transcription activation function and the DNA binding domain of transcriptional enhancer factor-1. EMBO J 1993; 12: 2337-2348.

14. Montano MM, Lim RW. Glucocorticoid effects on the skeletal muscle differentiation program: analysis of clonal proliferation, morphological differentiation and the expression of muscle-specific and regulatory genes. Endocr Res 1997; 23: 37-57.
15. Anderson $\mathrm{C}$, Catoe $\mathrm{H}$, Werner R. MIR-206 regulates connexin43 expression during skeletal muscle development. Nucleic Acids Res 2006; 34: 5863-5871.

16. Guo K, Wang J, Andres V, Smith RC, Walsh K. MyoD-induced expression of p21 inhibits cyclin-dependent kinase activity upon myocyte terminal differentiation. Mol Cell Biol 1995; 15: $3823-3829$

17. Halevy O, Novitch BG, Spicer DB, Skapek SX, Rhee J, Hannon GJ et al. Correlation of terminal cell cycle arrest of skeletal muscle with induction of p21 by MyoD. Science 1995; 267: 1018-1021.

18. Sugiura K, Muro Y, Futamura K, Matsumoto K, Hashimoto N, Nishizawa $Y$ et al. The unfolded protein response is activated in differentiating epidermal keratinocytes. $J$ Invest Dermatol 2009; 129: 2126-2135.

19. Nakanishi $K$, Sudo $T$, Morishima N. Endoplasmic reticulum stress signaling transmitted by ATF6 mediates apoptosis during muscle development. J Cell Biol 2005; 169: 555-560.

20. Acosta-Alvear D, Zhou Y, Blais A, Tsikitis M, Lents NH, Arias C et al. XBP1 controls diverse cell type- and condition-specific transcriptional regulatory networks. Mol Cell 2007; 27: 53-66.

21. Yoshida H, Matsui T, Yamamoto A, Okada T, Mori K. XBP1 mRNA is induced by ATF6 and spliced by IRE1 in response to ER stress to produce a highly active transcription factor. Cell 2001; 107: 881-891.

22. Lee K, Tirasophon W, Shen X, Michalak M, Prywes R, Okada T et al. IRE1-mediated unconventional mRNA splicing and S2P-mediated ATF6 cleavage merge to regulate XBP1 in signaling the unfolded protein response. Genes Dev 2002; 16: 452-466.

23. Harding HP, Calfon M, Urano F, Novoa I, Ron D. Transcriptional and translational control in the Mammalian unfolded protein response. Annu Rev Cell Dev Biol 2002 : 18: 575-599.

24. Sambasivan R, Cheedipudi S, Pasupuleti N, Saleh A, Pavlath GK, Dhawan J. The small chromatin-binding protein p8 coordinates the association of anti-proliferative and pro-myogenic proteins at the myogenin promoter. J Cell Sci 2009; 122: 3481-3491.

25. Vietor I, Vadivelu SK, Wick N, Hoffman R, Cotten M, Seiser C et al. TIS7 interacts with the mammalian SIN3 histone deacetylase complex in epithelial cells. Embo J 2002; 21: 4621-4631.

26. Vadivelu SK, Kurzbauer R, Dieplinger B, Zweyer M, Schafer R, Wernig A et al. Muscle regeneration and myogenic differentiation defects in mice lacking TIS7. Mol Cell Biol 2004; 24: 3514-3525

27. Cao X, Pfaff SL, Gage FH. YAP regulates neural progenitor cell number via the TEA domain transcription factor. Genes Dev 2008; 22: 3320-3334.

28. Cao Y, Yao Z, Sarkar D, Lawrence M, Sanchez GJ, Parker MH et al. Genome-wide MyoD binding in skeletal muscle cells: a potential for broad cellular reprogramming. Dev Cell 2010; 18: 662-674.

29. Aziz A, Liu QC, Dilworth FJ. Regulating a master regulator: establishing tissue-specific gene expression in skeletal muscle. Epigenetics 2010; 5: 691-695.

30. Sok J, Wang XZ, Batchvarova N, Kuroda M, Harding H, Ron D. CHOP-Dependent stressinducible expression of a novel form of carbonic anhydrase VI. Mol Cell Biol 1999; 19: 495-504.

31. Goruppi S, Patten RD, Force T, Kyriakis JM. Helix-loop-helix protein p8, a transcriptional regulator required for cardiomyocyte hypertrophy and cardiac fibroblast matrix metalloprotease induction. Mol Cell Biol 2007; 27: 993-1006.

32. Kong DK, Georgescu SP, Cano C, Aronovitz MJ, lovanna JL, Patten RD et al. Deficiency of the transcriptional regulator $p 8$ results in increased autophagy and apoptosis, and causes impaired heart function. Mol Biol Cell 2010; 21: 1335-1349.

33. Nishioka N, Inoue K, Adachi K, Kiyonari H, Ota M, Ralston A et al. The Hippo signaling pathway components Lats and Yap pattern Tead4 activity to distinguish mouse trophectoderm from inner cell mass. Dev Cell 2009; 16: 398-410.

34. Sawada A, Kiyonari H, Ukita K, Nishioka N, Imuta Y, Sasaki H. Redundant roles of Tead1 and Tead2 in notochord development and the regulation of cell proliferation and survival. Mol Cell Biol 2008; 28: 3177-3189.

35. Vial C, Zuniga LM, Cabello-Verrugio C, Canon P, Fadic R, Brandan E. Skeletal muscle cells express the profibrotic cytokine connective tissue growth factor (CTGF/CCN2), which induces their dedifferentiation. J Cell Physiol 2008; 215: 410-421.

36. Watt KI, Judson R, Medlow P, Reid K, Kurth TB, Burniston JG et al. Yap is a novel regulator of $\mathrm{C} 2 \mathrm{C} 12$ myogenesis. Biochem Biophys Res Commun 2010; 393: 619-624.

37. Chen HH, Maeda T, Mullett SJ, Stewart AF. Transcription cofactor Vgl-2 is required for skeletal muscle differentiation. Genesis 2004; 39: 273-279.

38. Delacroix L, Moutier E, Altobelli G, Legras S, Poch O, Choukrallah MA et al. Cell-specific interaction of retinoic acid receptors with target genes in mouse embryonic fibroblasts and embryonic stem cells. Mol Cell Biol 2010; 30: 231-244.

39. Trapnell C, Williams BA, Pertea G, Mortazavi A, Kwan G, van Baren MJ et al. Transcript assembly and quantification by RNA-Seq reveals unannotated transcripts and isoform switching during cell differentiation. Nat Biotechnol 2010; 28: 511-515.

40. Anders S, Huber W. Differential expression analysis for sequence count data. Genome Biol 2010; 11: R106.

\section{Supplementary Information accompanies the paper on Cell Death and Differentiation website (http://www.nature.com/cdd)}

